\title{
Astronomical Education for public and its future development in Mongolia
}

\author{
R. Tsolmon, V. Oyudari and A. Dulmaa \\ NUM-ITC-UNESCO Space Science and Remote Sensing Laboratory ,National University of \\ Mongolia email: tzr112@psu.edu, tsolmon91@yahoo.com
}

International activities for astronomy began when Mongolia joined the IAU at the General Assembly held in Prague in August 2006, because space scientists, astronomers and researchers in Mongolia are coming to understand that astronomy can help Mongolian socioeconomic development. For instance, astronomy can increase general interest and encourage public engagement in the sciences.

Mongolia is the only one of the ancient nomad states to retain the tenets of its original nomadic civilization, including the classic migration of livestock and closeness to nature. Like other Asian nations, the astronomy has been developing since ancient times as a science for Mongols with deep sacral worship of the blue sky and eternal heavens.

Today, Mongolia does not have a planetarium for public viewing. Since the collapse of the communist system due to economic difficulties, little attention has been paid to science and astronomy education. There is a lack of training opportunities for Astronomy amateurs and the public. The educational system of Mongolia has no astronomy textbook in Mongolian language. Astronomy education falls even further behind other developing countries. If we will have planetarium the younger generation as well as the general public will find planetariums fascinating. We hope that IAU will help us to raise awareness by giving us the means to provide a public planetarium. We desperately need more research opportunities and education in astronomy in Mongolia. Mongolia has a climate most conducive for observational astronomy, has a good advantageous to observe moon and stars the air is mostly dry and industrial and light pollution are mainly almost absent. In order to develop astronomy and space science in Mongolia we need many years of cooperation with international educational organizations in Asian region.

For developing graduate education and training programs we should involve international school and activities with help international astronomy community. Taking advantage of Mongolia's dry climate, comparatively less light pollution that promise precise chances of astronomical research and study we aim to bring science of astronomy to new qualitative level of development. Thus it creates a demand for new astronomers in Mongolia. 